\title{
A distant candle
}

\section{The discovery of a supernova at a redshift of one-half holds the promise of a new determination of the cosmic density parameter. But promise and practice are some way apart.}

DECIDING whether the Universe will expand forever or instead one day fall back on itself is very easy. All one needs to do is find two objects of identical luminosity, one nearby and one farther away, and measure their apparent brightnesses. For uniform cosmological models in which the single unknown parameter is $\Omega$, the ratio of density to the critical density required for closure, there is a family of curves relating brightness to redshift, so the accurate measurement of two points on that curve suffices to pick out one value of $\Omega$. If it is less than one, the Universe expands forever; if it is greater than one, the Universe must eventually collapse.

Astrophysical objects of fixed brightness are not easy to find, but there is one good possibility. A type Ia supernova occurs when a white dwarf blows up, a catastrophe triggered by the gradual accretion of material from a companion star onto the surface of the white dwarf until the internal density rises to the point where gravitational pressure overwhelms the ability of the degenerate core to withstand it. A thermonuclear event ensues, blowing the white dwarf to pieces, and the nature of this explosion ought to depend on the physics of white dwarfs and little else. A type la supernova ought therefore to be the same no matter where it occurs, so that it can reliably be called a "standard candle", of the sort that cosmologists have long hoped to find.

This is all well known, but its significance has come to the fore lately because of the discovery, by Saul Perlmutter and colleagues at the Lawrence Berkeley Laboratory, of a type Ia supernova in an anonymous galaxy whose redshift was subsequently established, by Karl Glazebrook of the University of Durham, to be $z=0.457$. This is the most distant supernova yet seen, and the hope is that it will act as a calibration point on the cosmological brightness versus redshift curve at a large enough redshift that curves for different values of $\Omega$ (say, 0.2 and 1.0) will be easily distinguished.

Simple enough but, as always with cosmological measurements, things are not as simple as they seem. The fact that this object is called a type Ia supernova implies, of course, that there are other types of supernova, so the first thing for Perlmutter and colleagues to establish is that the object they have seen is what they think it is. Type I supernovae are distinguished from Type II by the absence of hydrogen lines in the spectrum, which is easy enough to check, but type I supernovae are then split into different sorts by more subtle spectral analy-

NATURE · VOL 360 - 3 DECEMBER 1992 sis. Type Ib supernovae lack a certain $\mathrm{Si}$ absorption line and, at late stages of development, are dominated by oxygen rather than iron features, but in early outburst can be hard to distinguish from Type Ia supernovae. They are, however, substantially dimmer, so an astronomer mistaking a type $\mathrm{Ib}$ for a type Ia would think the Universe much bigger than it is.

Supposing the supernova in question to have been correctly labelled as a type Ia, there are still plenty of shoals to navigate. By the brightness of a supernova, which is the number the cosmological brightnessredshift curve has to pass through, is generally meant the peak brightness, which occurs a few days after outburst. Perlmutter et al. did not catch their distant supernova at its peak, but have been tracking its falling brightness and attempting to extrapolate thereby when the peak occurred and how bright it was. But any such extrapolation is reliable only to the extent that this type Ia is like every other type Ia, and it is precisely this kind of assumption one would like to avoid, as far as possible, in this exercise.

Moreover, local type Ia supernova brightness curves are most similar, and best studied, in the optical B-band (a blue part of the spectrum), whereas the supernova at $\mathrm{z}=$ 0.457 has been measured in the infrared Rband. Light curves are not identical in different wavelength bands, and there is an offset in the moment of peak brightness in different bands. But here is a piece of luck: the R-band, for an object at a redshift of about one-half, is equivalent to the B-band at the source, so that Perlmutter et al. are in fact measuring precisely the light-curve they need to measure to make the best comparison with the body of evidence on local type Ia supernovae.

Set against this, however, is the problem that the observed colours of any astronomical object are modified - reddened - by dust and gas in its local environment. If it were true that type Ia supernovae are precisely identical, then the effects of reddening could be subtracted out by comparing observations in different wavelength bands. But Perlmutter et al. have only a partial supernova lightcurve at a single wavelength, and they want moreover to avoid undue reliance on the assumption that their supernova is the same as everyone else's. In local supernova surveys it is possible, for example, to include only events that occur in elliptical galaxies, for which reddening due to dust is presumably slight, but if one is interested in supernovae at moderate redshifts, asking for a precise identification of the type of galaxy they are in is asking for a lot.

Setting these mere observational difficulties aside, there remains the most vexing question of all: how identical are type Ia supernovae? To say that they happen when a white dwarf reaches a certain critical core density implies that they should all be the same, but the empirical fact is that local type Ia supernovae are not precisely the same, even allowing for observational uncertainties. The colours at maximum vary slightly, and there are some 'peculiar' type Ia events, which show a variety of odd photometric and spectroscopic features.

This is hardly unreasonable. White dwarfs are a homogeneous class of objects, compared, for example, to main sequence stars, but they can have slightly different masses, interior temperatures, compositions, rotation rates and so on. All of these factors may alter, in subtle ways, the nature of a white dwarf explosion. Compositional differences, because they pertain mostly to the outer layers of the white dwarf, may have a marked effect on the light-curve near-peak brightness, because one is then observing the outermost edge of the ejected material. Theoretical models of type Ia explosions suggest two broad possibilities: the white dwarf can be dispersed by a nuclear deflagration wave or by a supersonic detonation wave, the time-scale and energy release being different between the two cases.

What this all means, as Perlmutter et al. certainly recognise, is that many moderateredshift type la supernovae will have to be detected. The optimistic view is that when 20 or 30 such events have been recorded, they will cluster around a single cosmological brightness-redshift curve in such a way that all the scatter can be put down to observational uncertainties; this will imply that all type Ia supernovae are sufficiently similar for a value of $\Omega$ to be determined. The pessimistic view is that there is some inescapable systematic variation in the characteristics of type Ia supernovae, so that as Perlmutter et al. search at higher redshift they will preferentially detect the intrinsically brighter events, which will not be a truly representative subsample of the whole population. And this will create a bias in their estimate of $\Omega$, which is the problem observational cosmologists have been struggling with for decades. But the only way to find out what will happen is to undertake the necessary observations, and that is what Perlmutter et al. are undoubtedly planning to do.

David Lindley 